\title{
Bile acid toxicity in Paneth cells contributes to gut dysbiosis induced by high-fat feeding
}

Hui Zhou, ${ }^{1,2}$ Shi-Yi Zhou, ${ }^{1}$ Merritt Gillilland III, ${ }^{1}$ Ji-Yao Li, ${ }^{1}$ Allen Lee, ${ }^{1}$ Jun Gao, ${ }^{1}$ Guanpo Zhang, ${ }^{1,3}$ Xianjun $\mathrm{Xu}^{2}{ }^{2}$ and Chung Owyang ${ }^{1}$

'Division of Gastroenterology and Hepatology, Department of Internal Medicine, University of Michigan Health System, Ann Arbor, Michigan, USA. ${ }^{2}$ Department of Gastroenterology, Shanghai General Hospital, Shanghai Jiao Tong University School of Medicine, Shanghai, China. ${ }^{3}$ Department of Gastroenterology, 900 Hospital of the Joint Logistics Team, Fuzhou, China.

High-fat feeding (HFF) leads to gut dysbiosis through unclear mechanisms. We hypothesize that bile acids secreted in response to high-fat diets (HFDs) may act on intestinal Paneth cells, leading to gut dysbiosis. We found that HFF resulted in widespread taxonomic shifts in the bacteria of the ileal mucosa, characterized by depletion of Lactobacillus and enrichment of Akkermansia muciniphila, Clostridium XIVa, Ruminococcaceae, and Lachnospiraceae, which were prevented by the bile acid binder cholestyramine. Immunohistochemistry and in situ hybridization studies showed that G protein-coupled bile acid receptor (TCR5) expressed in Paneth cells was upregulated in the rats fed HFD or normal chow supplemented with cholic acid. This was accompanied by decreased lysozyme+ Paneth cells and $\alpha$-defensin 5 and 6 and increased expression of XBP-1. Pretreatment with ER stress inhibitor 4PBA or with cholestyramine prevented these changes. Ileal explants incubated with deoxycholic acid or cholic acid caused a decrease in $\alpha$-defensin 5 and 6 and an increase in XBP-1, which was prevented by TCR5 antibody or 4PBA. In conclusion, this is the first demonstration to our knowledge that TCR5 is expressed in Paneth cells. HFF resulted in increased bile acid secretion and upregulation of TCR5 expression in Paneth cells. Bile acid toxicity in Paneth cells contributes to gut dysbiosis induced by HFF.

Conflict of interest: The authors have declared that no conflict of interest exists.

Copyright: (c) 2020, Zhou et al. This is an open access article published under the terms of the Creative Commons Attribution 4.0 International License.

Submitted: April 29, 2020 Accepted: September 9, 2020 Published: October 15, 2020

Reference information: JCI Insight. 2020;5(20):e138881. https://doi.org/10.1172/jci. insight.138881.

\section{Introduction}

Chronic high-fat feeding (HFF) may induce gut dysbiosis and alter host homeostasis, resulting in mucosal inflammation and metabolic endotoxemia (1-3). However, little is known about how HFF induces gut dysbiosis. While HFF may contribute directly to gut dysbiosis $(4,5)$, it is also possible that alterations in gut bacterial communities may be mediated by host factors in response to high-fat diet (HFD).

HFF leads to an increase in circulating bile acids (6) and affects gut bacterial communities by changing the bile acid profile $(7,8)$. Bile acids recently have been reported to play a role in gut dysbiosis $(9-11)$. After ingestion of a meal, the primary bile acids (cholic acid [CA] and chenodeoxycholic acid) are secreted into the duodenum, where they facilitate fat absorption. Normally, more than $95 \%$ are efficiently absorbed from the terminal ileum and transported back to the liver via the enterohepatic circulation. Small quantities of primary bile acids reach the terminal ileum and colon and undergo deconjugation and dehydroxylation by certain gut bacteria to form secondary bile acids, deoxycholic acid (DCA) and lithocholic acid (LCA) (12). Bile acids, especially secondary forms such as DCA, have strong antimicrobial activity and cytotoxicity and may regulate the composition of gut bacterial communities and host physiology $(13,14)$ In addition to the direct antimicrobial actions, we explored other mechanisms by which bile acids may affect host factors and alter the gut bacterial composition

Both primary and secondary bile acids exert their effects by activating nuclear (15) and plasma membrane receptors $(16,17)$. G protein-coupled receptor TGR5 is a bile acid plasma membrane receptor that is widely distributed in the gastrointestinal tract (18). TGR5 regulates bile acid synthesis, intestinal secretion, glucose homeostasis, energy expenditure, gastrointestinal motility, and inflammation, while overstimulation has been linked to human diseases, such as esophageal and gastric carcinoma (19-23). Here, we hypothesis that TGR 5 receptors are present in Paneth cells. This may provide a mechanism by which bile acids regulate intestinal immune function. 
Paneth cells are highly specialized epithelial cells located at the base of the crypts of Lieberkühn of the small intestine. Paneth cells secrete antimicrobial peptides into the crypt lumen, which protect the host from enteric pathogens, help to shape the composition of the colonizing bacteria, safeguard against bacterial translocation across the epithelium, and act as immune modulators and trophic factors (24-26). On the other hand, Paneth cell dysfunction has been associated with gut bacterial changes in obese individuals (27). Recently, Zheng et al. reported that a HFD caused rapid and significant increases in the intestinal bile acid pool, accompanied by an alteration in bacterial composition (7). This raises the possibility that, in addition to the direct effect of bile acid on intestinal bacteria, bile acids may modulate gut immune function and indirectly alter gut bacterial communities. We hypothesized that bile acids elevated by HFF regulate intestinal Paneth cell function, which may contribute to gut dysbiosis in the ileal mucosa.

\section{Results}

TGR5 expression in the Paneth cells, and HFF increases bile acids and enhances TGR5 expression in ileal crypts. Immunohistochemical staining was performed using ileal specimens and isolated crypt cells. TGR5 expression was observed in the ileal crypts. Lysozyme staining was used to identify Paneth cells. Merged images show that TGR5 was expressed by Paneth cells (Figure 1A). These findings were confirmed in isolated crypt cells. A lysozyme ${ }^{+}$Paneth cell expressing TGR5 is shown in Figure 1B. Double in situ hybridization using dig-labeled lysozyme, a specific marker for Paneth cells, and $\left.{ }^{35} \mathrm{~S}\right]-$ labeled TGR5 confirmed that $78 \%$ of Paneth cells expressed TGR5 (Figure 1C).

Rats fed a control diet of regular chow (RC, $11 \% \mathrm{kcal}$ from fat) had a fecal bile acid level of $1.3 \pm 0.1$ $\mu \mathrm{mol} / \mathrm{g}$. A 2-week HFF (58\% kcal from fat) increased the fecal bile acid level to $2.1 \pm 0.2 \mu \mathrm{mol} / \mathrm{g}(n=6$, $P<0.05$ compared with RC; Figure 1D). A similar increase was observed in rats fed a HFD supplemented with cholestyramine (CHO) $(2.6 \pm 0.2 \mu \mathrm{mol} / \mathrm{g} ; n=6, P<0.01$ compared with RC; Figure 1D). Rats fed RC had a serum bile acid level of $19.6 \pm 0.6 \mu \mathrm{mol} / \mathrm{L}$ after a 12-hour fast. A 2-week HFF increased the fasting serum bile acid level to $28.4 \pm 2.0 \mu \mathrm{mol} / \mathrm{L}(n=6, P<0.01$; Figure $1 \mathrm{D})$. A similar increase was observed in rats fed RC supplemented with CA (44.4 $\pm 1.4 \mu \mathrm{mol} / \mathrm{L} ; n=6, P<0.01$; Figure 1D). Serum bile acid levels were normalized by concurrent administration of $\mathrm{CHO}$ in rats on $\mathrm{HFD}(17.2 \pm 1.4 \mu \mathrm{mol} / \mathrm{L} ; n=6$, $P<0.01$; Figure 1D). These results indicate that HFF elevates fecal and serum bile acid level.

Compared with that in controls, there was a significant increase in TGR5 protein expression in ileal crypts from rats fed a HFD $(n=5, P<0.05)$ or RC supplemented with CA $(n=4, P<0.05$; Figure $1 \mathrm{E})$. Cofeeding with CHO prevented the increase TGR5 in response to HFD ( $n=5, P<0.01$; Figure 1E).

HFF induces a decrease in Paneth cells and antimicrobial peptides, which is prevented by cofeeding with $\mathrm{CHO}$. Using CD45 as a hematopoietic cell marker, Paneth cells were identified as a CD45-1ysozyme ${ }^{+}$cell fraction. Flow cytometry showed a reduction in lysozyme ${ }^{+}$Paneth cells $(n=6, P=0.05$; Figure 2A) as well as lysozyme ${ }^{+}$TGR $^{+}$Paneth cell expression $(n=6, P<0.01$; Figure $2 \mathrm{~A})$ in the crypts after HFF or oral CA feeding ( $n=5-6, P<0.01$; Figure 2A). Administration of $\mathrm{CHO}$ prevented this decrease in lysozyme ${ }^{+}$ Paneth cells although it did not affect the fraction expressing TGR5 ( $n=6, P<0.01$; Figure 2A).

Transmission electron micrograph of Paneth cells showed a loss of electron-dense secretory granules and an increase in the percentage of granules with vacuoles in the ileum of rats given a HFD (Figure $2 \mathrm{~B}$ ) or rats fed $\mathrm{RC}$ supplemented with $\mathrm{CA}$ (Figure $2 \mathrm{~B}$ ). These changes were prevented by cofeeding with $\mathrm{CHO}$ in rats given a HFD (Figure 2B).

RT-qPCR studies demonstrated that HFF caused a $68 \%$ and $67 \%$ decrease in gene expression of $\alpha$-defensin 5 (Defa5) and $\alpha$-defensin 6 (Defab) in the crypts of ileum, respectively ( $n=5$, both $P<0.01$, Figure 2C). These changes were prevented by concurrent feeding with $\mathrm{CHO}(n=5$, both $P<0.01$, Figure 2C). A similar reduction in Defa5 and Defa6 was observed in rats fed with CA (data not shown).

HFF induces protein and gene expression of ER stress, autophagy, and DNA damage. Using X-box-binding protein 1 (XBP-1) as an ER stress marker, we performed immunohistochemical studies and showed that XBP-1 expression was increased at the bottom of ileal crypts of rats given a HFD (Figure 3A). RT-qPCR studies showed increased gene expression of XBP-1 and autophagy-related protein 16-1 (ATG16L1, an autophagy marker) in the crypts of ileum after 2 weeks of $\operatorname{HFF}(n=5$, both $P<0.01)$. The gene expression of $A T G 16 L 1$ and poly ADP ribose polymerase (PARP, an apoptotic marker) increased in rats fed CA ( $n=$ 4-5, $P<0.01$; Figure 3B). Concurrent feeding with $\mathrm{CHO}$ prevented these increases in $X B P-1, A T G 16 L 1$, and $P A R P 1$ gene expression ( $n=5-6, P<0.05$ or $P<0.01$; Figure $3 \mathrm{~B}$ ). To demonstrate that these cellular changes in Paneth cells are due to ER stress, we showed that administration of 4-phenylbutyrate (4PBA), 
A

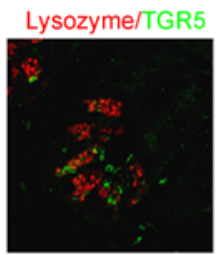

DAPI

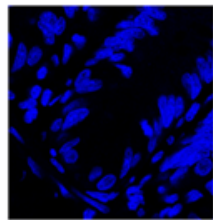

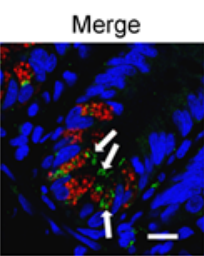

B

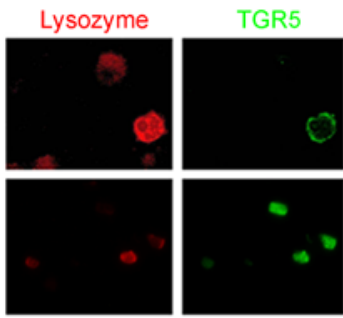

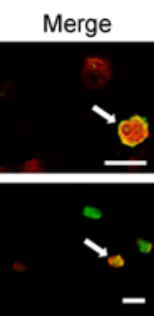

C Antisense probes dig-lysozyme(purple color)+ ${ }^{35} \mathrm{~S}-T$ TR5 (silver grains)

Sense probes

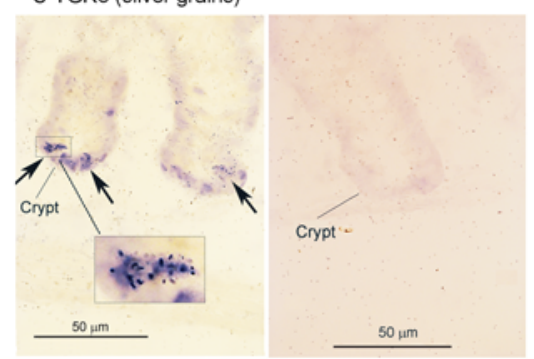

E

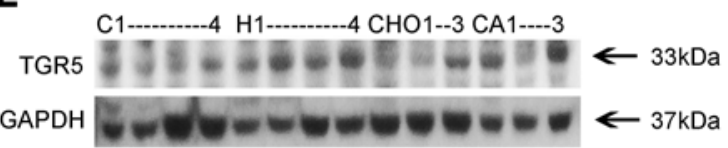

D

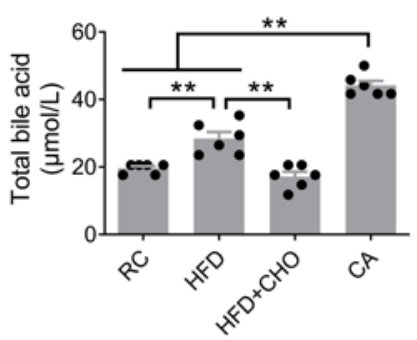

Feces

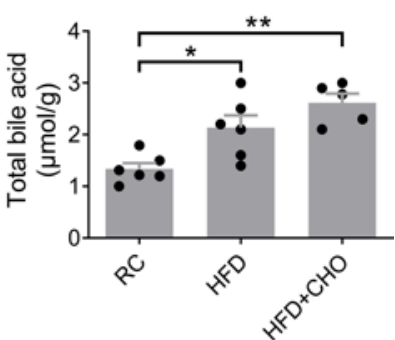

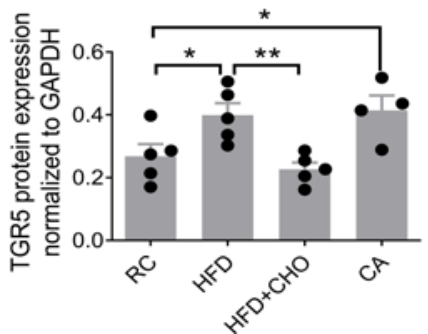

Figure 1. TGR5 expression in the Paneth cells; HFD increases bile acids and enhances TGR5 expression in ileal crypts. (A and B) Immunocytochemical staining using sections of ileal specimens and isolated crypt cells showed that TCR5 expression was observed in the lysozyme ${ }^{+}$Paneth cells (white arrows) in ileal crypts. Scale bar: $10 \mu \mathrm{m}$. (C) Left: hybridization using antisense probes. Purple represents lysozyme mRNA, a specific marker for Paneth cells, which are located at the bottom portion of crypt; silver grains indicate TGR5 mRNA. Arrows indicate colocalization. Right: Dig-lysozyme and [ ${ }^{35}$ S]-TGR5 sense probes were used as the negative control. Note that TGR5 receptors are also expressed in other cell types in the intestinal crypts. Scale bar: $50 \mu \mathrm{m} ; 3-\mathrm{fold}$ magnification (inset). (D) Measurement of total bile acid concentration. A 2-week high-fat diet (HFD) induced a $45 \%$ increase in serum and $60 \%$ increase in fecal bile acid compared with control (RC) $(n=5-6)$. The increased total serum bile acid was prevented by concurrent administration of cholestyramine $(n=6)$. A similar increase in serum bile acid was observed in cholic acid group $(n=6)$. (E) The density of the TCR5-immunoreactive band at $33 \mathrm{kDa}$ was observed in the ileal crypts of rats given HFD. TGR5 protein expression increased in ileal crypts of HFD rats compared with control ( $n=5$ ). This increase was prevented by concurrent administration of cholestyramine $(n=5)$. A similar increase was also observed in rats with cholic acid $(n=4-5)$. Each bar represents mean \pm SEM. C, RC; H, HFD; CHO, HFD + cholestyramine; CA, cholic acid. $P$ values were determined by $1-$ way $A N O V A$. ${ }^{*} P<0.05$, ${ }^{*} P<0.01$.

an ER stress inhibitor, normalized $\alpha$-defensin expression and prevented induction of ER stress and autophagy caused by $\operatorname{HFF}(n=6, P<0.05$ or $P<0.01$; Figure 3B).

Western blot showed that the density of ATG16L1- (autophagy marker), BiP- (ER stress marker), and caspase-3-immunoreactive (cell apoptosis marker) bands at $60 \mathrm{kDa}, 75 \mathrm{kDa}$, and $19 \mathrm{kDa}$, respectively, were observed in the ileal crypts of rats fed RC. After HFF there were significant increases in ATG16L1- and caspase-3-immunoreactive bands $(n=5-6, P<0.05$ or $P<0.01$; Figure $3 C)$. Oral feeding with CA caused a similar increase in BiP and ATG16L1 expression ( $n=3-6, P<0.05$ or $P<0.01$; Figure 3C). These increases in $\mathrm{BiP}$, ATG16L1, and caspase-3 expression in response to HFF were prevented by concurrent feeding with $\mathrm{CHO}(n=4-6, P<0.05$ or $P<0.01$; Figure $3 \mathrm{C})$.

Ex vivo study (intestinal explants). To provide direct evidence that bile acids induce damage in Paneth cells, we showed that there was a decrease in Defa 5 and Defa6 gene expression and an increase in $X B P-1$ gene expression in whole-mount ileal tissue incubated with DCA $(100 \mu \mathrm{M})$ (Figure $4 \mathrm{~A})$ or CA $(100 \mu \mathrm{M})$ (Figure 4B) for 24 hours. These alterations were prevented by TGR5 antibody $(4 \mu \mathrm{g} / \mathrm{mL})$ or by 4PBA $(10 \mathrm{mM})$. However, administration of bile acid nuclear receptor, farnesoid $\mathrm{X}$ receptor (FXR) agonist GW4064 did not affect the expression of Defa5, Defa6, XBP-1, and ATG16L1 in intestinal explants (data not shown).

HFF alters bacterial community composition of the ileal mucosa, which is prevented by CHO or $4 P B A$. Compared with controls, there was a significant reduction in the relative abundance of Firmicutes and an increase in 
A
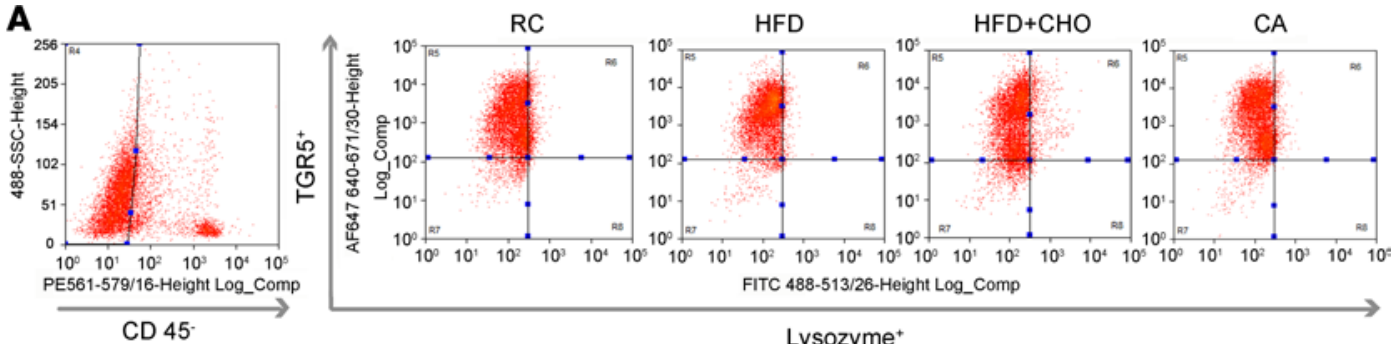

CD 45
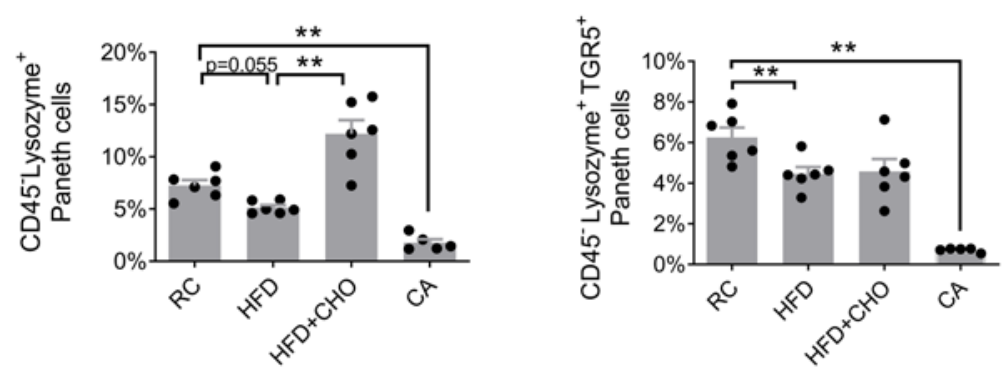

B
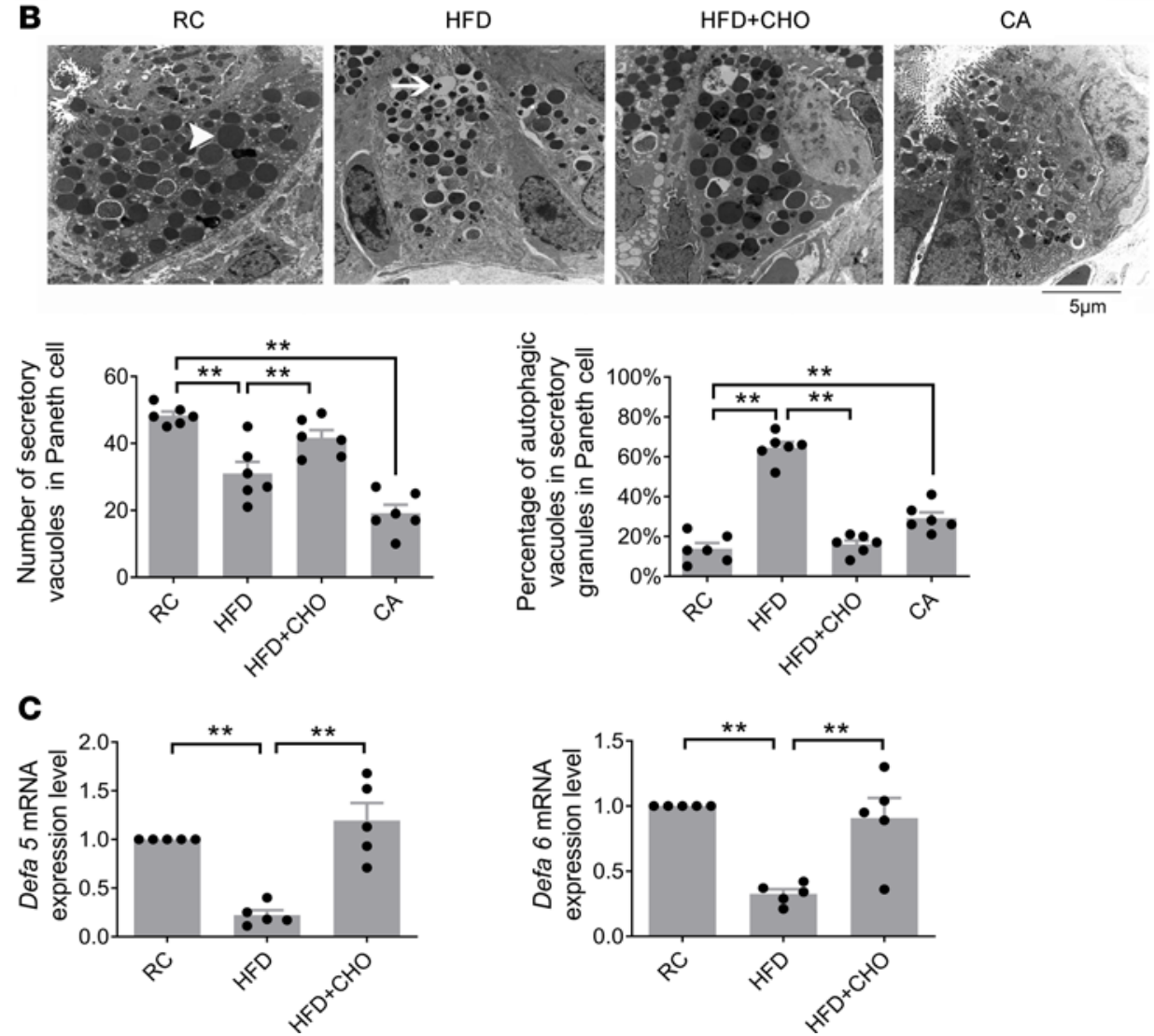

Figure 2. HFD induces a decrease in Paneth cells and antimicrobial peptide prevented by cofeeding with cholestyramine. (A) HFD induced a reduction in lysozyme ${ }^{+}$Paneth cells ( $n=6, P=0.055$ ) as well as lysozyme ${ }^{+}$TCR ${ }^{+}$Paneth cell expression $(n=6)$ in the crypts. Administration of cholestyramine prevented this decrease in lysozyme ${ }^{+}$Paneth cells $(n=6)$. Concurrent oral cholic acid also induced a significant decrease in both lysozyme ${ }^{+}$Paneth cells or lysozyme ${ }^{+}$GR5'Paneth cell $(n=5-6)$. (B) HFD

causes a decrease in Paneth cell secretory granules. Transmission electron micrograph of small intestinal Paneth cells showed a $34 \%$ and $53 \%$ decrease in Paneth cell secretory granules (white arrowhead) from rats fed a HFD and cholic acid diet, respectively $(n=6$, ${ }^{* *} P<0.01$ ). An increase in percentage of secretory granules with vacuoles (white arrow) was also observed in Paneth cell from rats fed a HFD, which was prevented by cofeeding with cholestyramine $(n=6)$. A similar increase in vacuoles was observed in rats given cholic acid $(n=6)$. Scale bar: $5 \mu \mathrm{m}$. (C) Reduction in gene expression of $\alpha$-defensin 5 (Defa5) and 6 (Defa6) in rats given a HFD. A 68\% and 67\% decrease in gene expression of Defa5 and Defa6, respectively, in the crypts of ileum in rats fed a HFD $(n=5)$. These decreases in Defa5 and Defa6 gene expression were prevented by concurrent oral feeding with cholestyramine $(n=5)$. CHO, cholestyramine. All data are shown as mean \pm SEM. $P$ values were determined by 1 -way ANOVA. ${ }^{* *} P<0.01$.

Verrucomicrobia after HFF (Figure 5, A and B). An increase in the relative abundance of Gram-negative bacteria after HFF and reduction of Gram-positive bacteria were observed (Figure 5, C and D). Among the Gram-negative bacteria, Verrucomicrobia (Akkermansia muciniphila) was significantly increased (Figure 5, B and E). Bacteroidetes showed an increase, but it did not reach statistical significance (Figure 5B). Specific taxa affected by HFF included a reduction in 4 taxa identified as Lactobacillus as well as enrichment in other operational taxonomic units (OTUs), such as Clostridium XIVa, Ruminococcaceae, Lachnospiraceae, and Akkermansia (linear discriminant analysis effect size [LEfSe], $P<0.05$, Figure 6, A and B). HFF resulted in a significant depletion in the relative abundance of Lactobacillaceae from $12.4 \%$ to $0.2 \%$ and an increase in the abundance of Verrucomicrobiaceae from 2.1\% to $18.9 \%$ (Figure 6D). 
A

Lysozyme

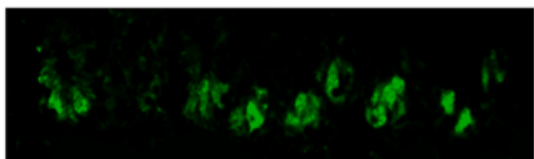

XBP-1

Merge
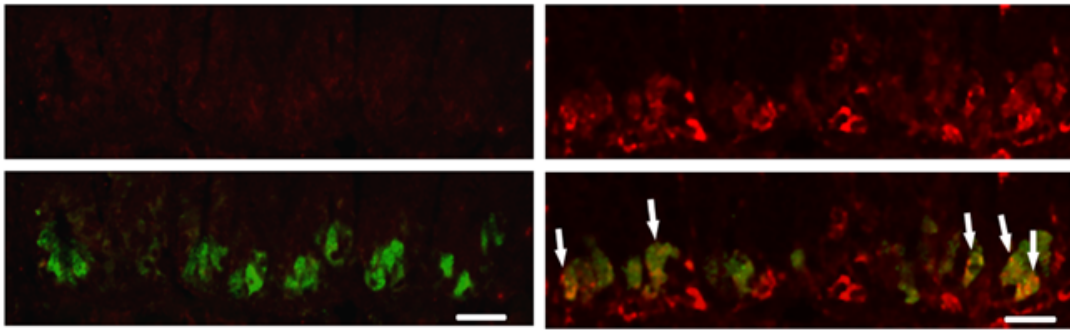

B

XBP-1

ATG16L1
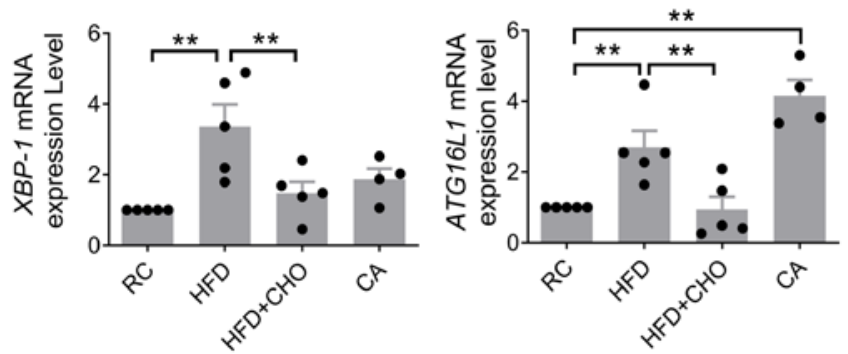

Defa 6
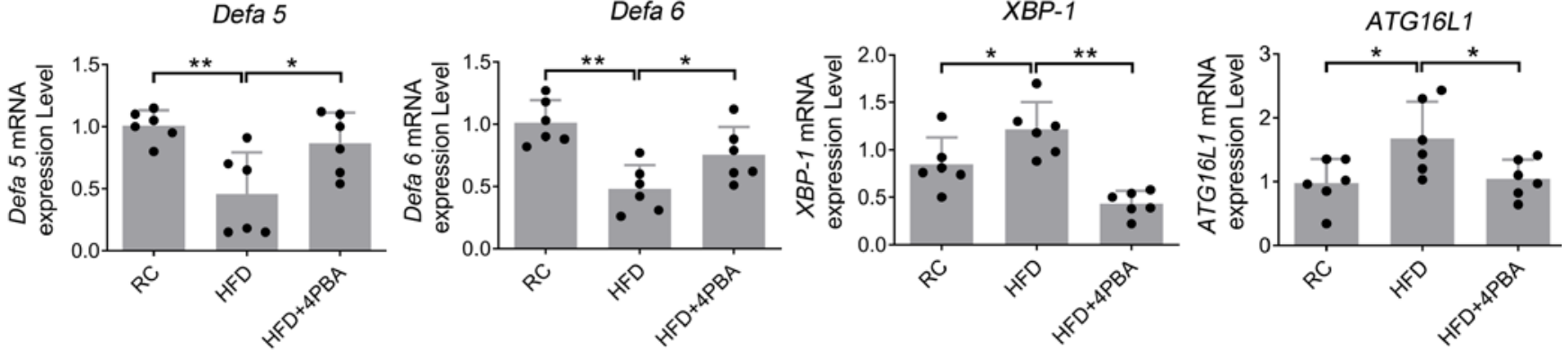

PARP 1

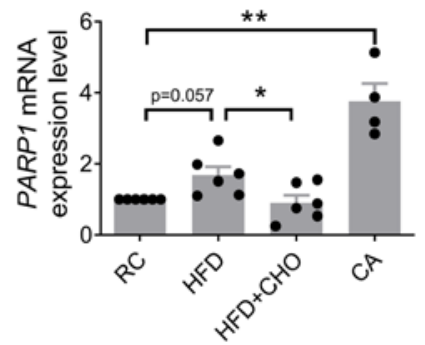

$X B P-1$

C C1 $-4 \mathrm{H} 1-$ $-4 \mathrm{CHO} 1$ -3 CA1-
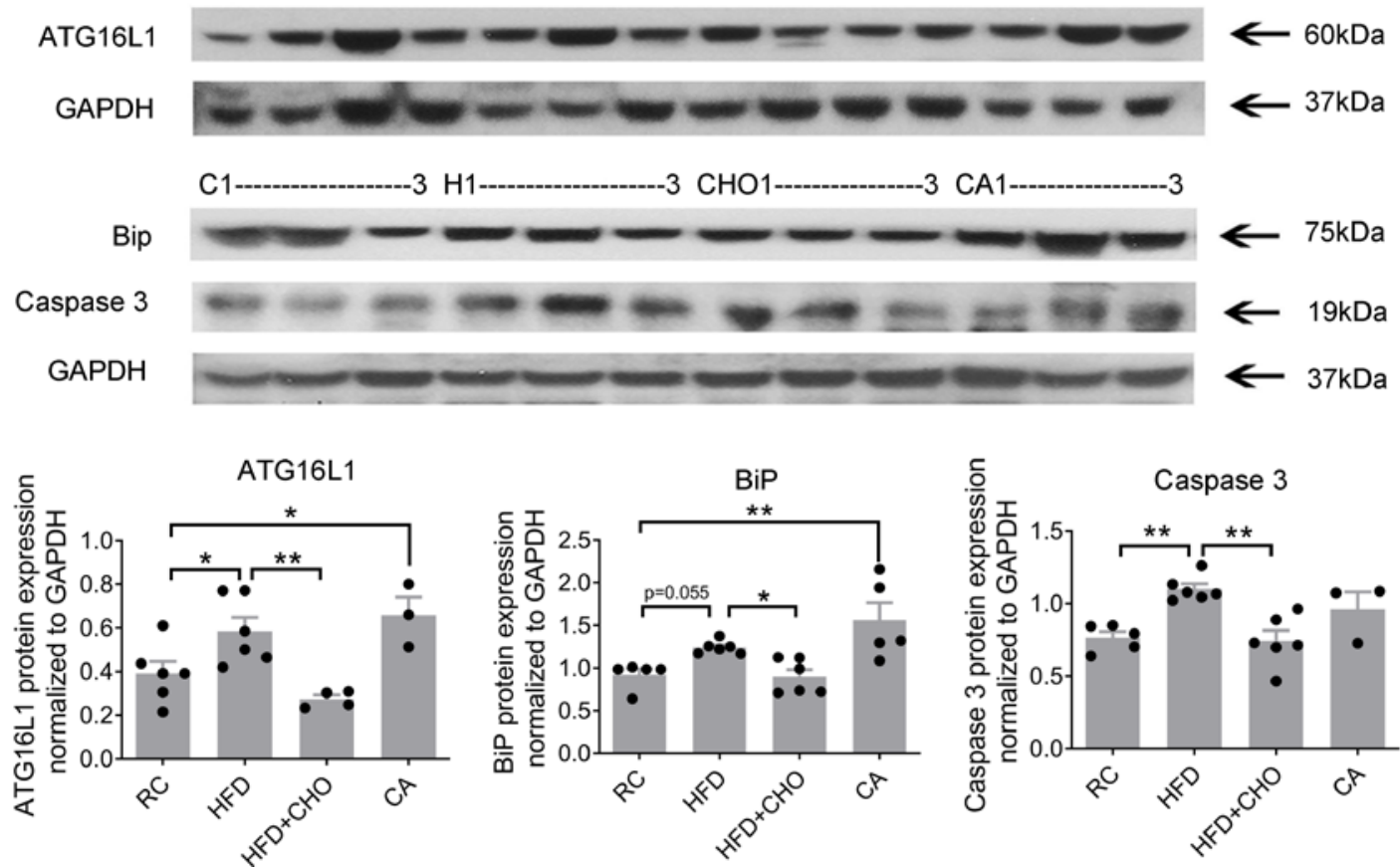
Figure 3. HFD induces protein and gene expression of ER stress, autophagy, and DNA damage. (A) Immunocytochemical staining of ileal specimen from rats fed regular chow (RC) or HFD (lysozyme, green; XBP-1 [ER stress marker], red). HFD induces increased expression of XBP-1 in lysozyme Paneth cells. Scale bar: $50 \mu \mathrm{m}$. (B) High-fat feeding caused an increase in gene expression of XBP-1 and ATC16L1 in the crypts of ileum $(n=5)$. These increases in $X B P-1$ and ATG16L1 gene expression were prevented by concurrent oral feeding with cholestyramine $(n=5)$. Oral feeding with cholic acid caused a similar increase in ATC16L1 and PARP1 gene expression $(n=4-5)$. HFD induced alteration of $\alpha$-defensins, autophagy, and ER stress were prevented by an ER stress inhibitor (4BPA) $(n=6)$ ). (C) Western blot showing the density of ATG16L1 (autophagy marker), BiP (ER stress marker), and caspase-3 (DNA damage marker) immunoreactive bands at $60 \mathrm{kDa}, 75 \mathrm{kDa}$, and $19 \mathrm{kDa}$, which were observed in the ileal crypts from rats fed RC. HFD caused a significant increase in ATC16L1 and caspase-3-immunoreactive bands $(n=5-6)$. Oral feeding with cholic acid caused a similar increase in ATC16L1 and BiP expression $(n=3-6)$. These increases in BiP, ATC16L1, and caspase-3 expression in response to HFD were prevented by concurrent oral feeding with cholestyramine $(n=4-6)$. Part of the membrane used for protein expression studies of TCR5 in Figure 1E was used for Western blotting of ATC16L1. Hence, the 2 blots share the same CAPDH. C, RC; H, HFD; CHO, HFD + cholestyramine; CA, cholic acid. $P$ values were determined by 1 -way ANOVA. ${ }^{*} P<0.05,{ }^{*} P<0.01$.

Concurrent oral feeding with the bile acid binder $\mathrm{CHO}$ significantly increased the relative abundance of Lactobacillaceae while depleting Verrucomicrobiaceae to baseline levels (Figure 6D). Further, Clostridium XI, Clostridium XIVa, and Akkermansia were significantly depleted, while Lactobacillus, Clostridium XI, and Clostridium sensu stricto were enriched in HFD+CHO when compared with HFD (LEfSe, $P<0.05$; Figure 6, B-D).

In a separate group of rats, gut microbial changes induced by 2 weeks of HFF were prevented by an inhibitor of ER stress, 4PBA (Figures 7 and Figure 8, A-C). Two OTUs, identified as Lactobacillus, were significantly depleted after HFF (Figure 8B). A number of OTUs were significantly enriched after HFF, including Lachnospiraceae, Akkermansia, Porphyromonadaceae, Bacteroides, and unclassified Firmicutes (LEfSe, $P<0.05$, Figure 8B). As a group, the relative abundances of Porphyromonadaceae, Lachnospiraceae, and Ruminococcaceae significantly increased from $0.32 \%$ to $27.1 \%, 3.1 \%$ to $25.4 \%$, and $2.5 \%$ to $8.6 \%$, respectively, after HFF (Figure 8D).

Concurrent treatment with 4PBA significantly decreased the relative abundance of the Porphyromonadaceae, Lachnospiraceae, and the Ruminococcaceae (Figure 8D). Furthermore, Bacteroides, unclassified Firmicutes, Lachnospiraceae, and Porphyromonadaceae were all significantly depleted in HFD+4PBA when compared with HFD (LEfSe, $P<0.05$, Figure 8C).

HFD-associated gut metagenome is altered with genes involved in amino acid and energy metabolism. To better understand the biological significance of the alteration in the gut bacterial communities induced by HFF and 4PBA, we investigated the metabolic features of the resulting communities using phylogenetic investigation of communities by reconstruction of unobserved states (PICRUSt). PICRUSt is a prediction tool for inferring the potential metabolism of a given bacterial community from an OTU table using precomputed Kyoto Encyclopedia of Genes and Genomes (KEGG) pathway reference profiles. Compared with RC, HFD resulted in enrichment of many genes involved in amino acid and energy metabolism (Figure 9). There was a significant reduction $(P<0.01)$ in the expression of genes for Gram-positive cell wall synthesis (peptidoglycan biosynthesis) and an enrichment of genes responsible for synthesis of the Gram-negative cell wall (lipopolysaccharide biosynthesis) in response to HFD. These changes were prevented by 4PBA (Figure 9). These potential metabolic changes are in agreement with the gut microbiome composition alterations showing enrichment of Gram-negative bacteria following 2 weeks of HFF, and these changes were prevented by treatment with $4 \mathrm{PBA}$.

\section{Discussion}

Although there is accumulating evidence that HFF may alter the gut microbial composition, the mechanisms leading to these changes are not clearly understood. In this study, we demonstrated that TGR5 receptors are expressed by Paneth cells in intestinal ileal crypts. Rodents fed HFD or RC supplemented with CA had significantly increased fecal and serum bile acid levels, increased TGR5 protein expression, and decreased numbers of intestinal Paneth cells. This was accompanied by markers of Paneth cell dysfunction, including subcellular abnormalities in Paneth cell secretory granules, decreased expression of Paneth cell-specific $\alpha$-defensins (Defa5 and Defa6), and enhanced ER stress and autophagy.

We found that an important mucosal microbial change associated with HFF is an increase in Gramnegative bacteria and corresponding decrease in Gram-positive bacteria. These were accompanied by phyla-level changes, including a decreased abundance of Firmicutes and a relative enrichment in Bacteroidetes and Verrucomicrobia. Our results differ from those of prior studies that have documented an increased abundance of Firmicutes and decreased relative proportion of Bacteroidetes in response to a HFD (28, 29). However, these prior studies mainly evaluated fecal samples representative of the distal gut microbiota, while regional variations in environmental conditions, such as $\mathrm{pH}$, oxygen availability, and nutrient availability, promote 
A

Defa 5

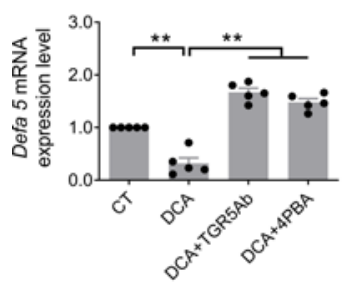

B

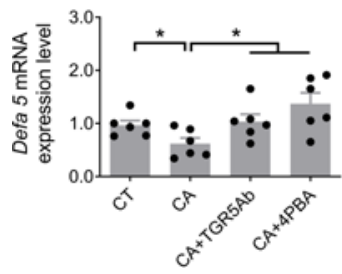

Defa 6

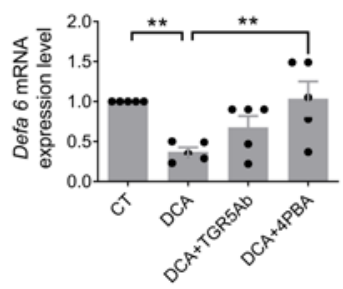

Defa 6

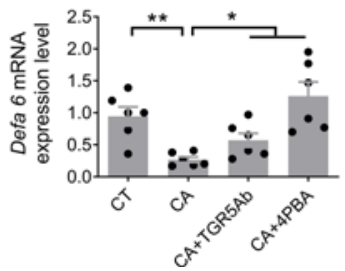

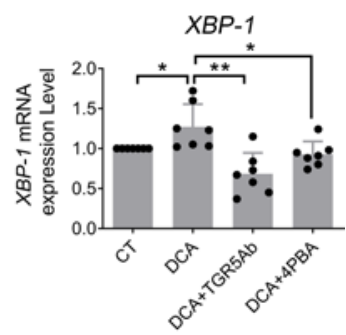

$X B P-1$

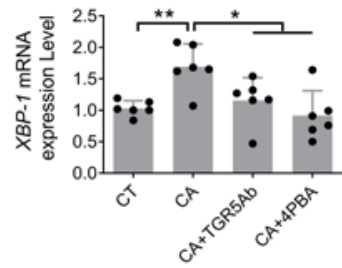

Figure 4. DCA and CA increase ER stress and reduction of $\boldsymbol{\alpha}$-defensins in cultured ileum (in vitro). (A) DCA (100 $\mu \mathrm{M})$ and (B) CA (100 $\mu \mathrm{M})$ caused a reduction in $\alpha$-defensin and ER stress in whole-mount cultured ileum. Pretreatment with TCR5 antibody $(4 \mu \mathrm{g} / \mathrm{mL})$ or 4 PBA $(10 \mathrm{mM})$ prevented the DCA- or CA-induced alteration of $\alpha$-defensins and ER stress $(n=5-7)$. $P$ values were determined by 1-way ANOVA. ${ }^{*} P<0.05,{ }^{*} P<0.01$.

differences in gut microbial ecology in different regions of the gastrointestinal tract (30). Similar to our findings, Lecomte et al. reported a decrease in abundance of Gram-positive Firmicutes and an increase in Gramnegative Bacteroidetes after HFF (29). These investigators used samples collected from the cecum, which is more closely aligned with our approach. Moreover, as opposed to luminal bacterial communities, which are

A
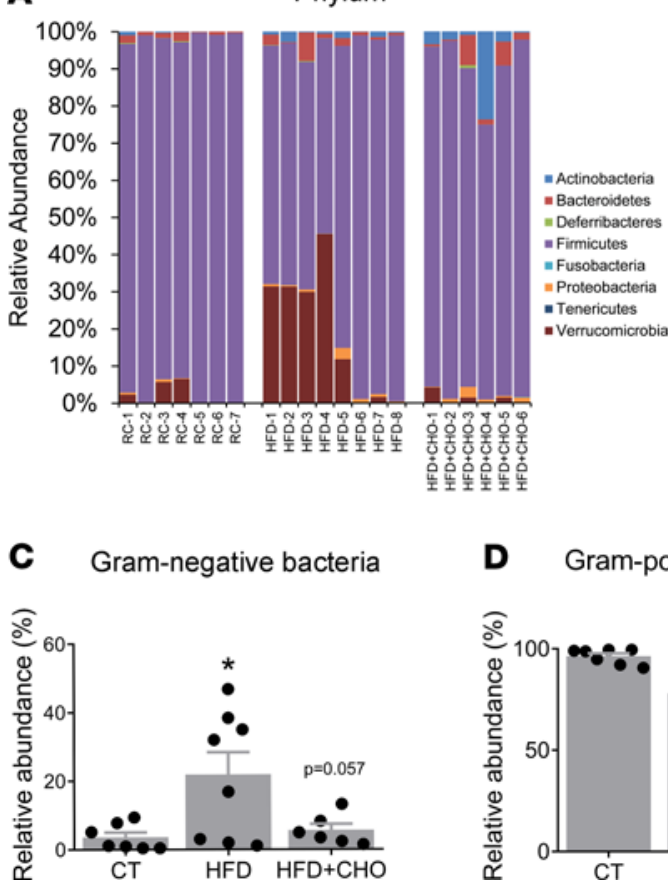

B

Firmicutes

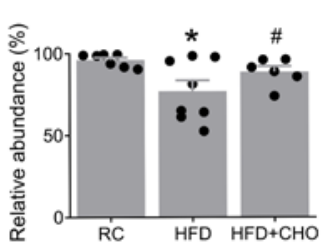

Verucomicrobia
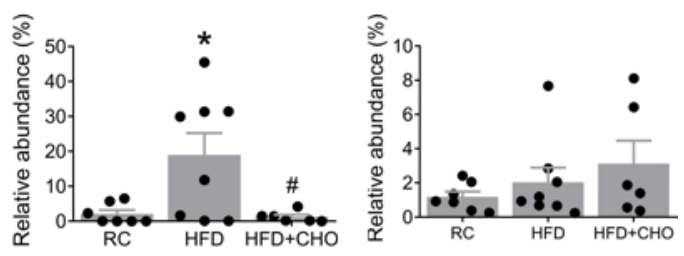

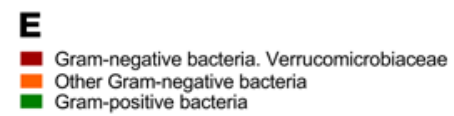

D Gram-positive bacteria
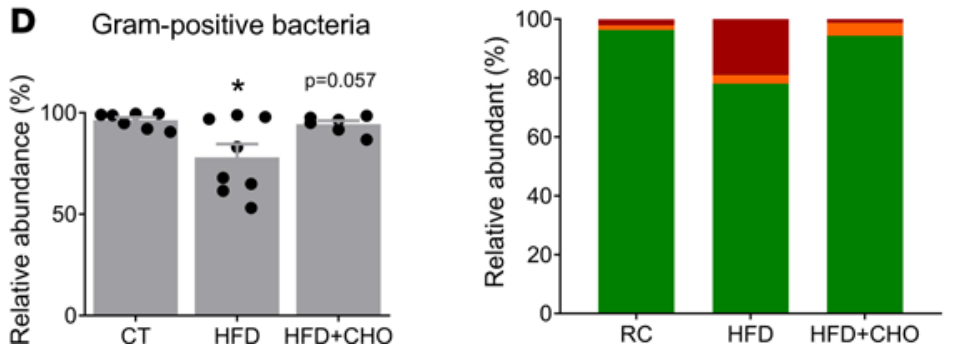

Figure 5. HFD-induced dysbiosis at the phylum level in the ileum mucosa is prevented by cholestyramine. (A) Relative abundance of OTUs classified at the level of phylum. (B) Relative abundance of Firmicutes, Verrucomicrobia, and Bacteroidetes. (C and D) Relative abundance of (C) Gram-negative and (D) -positive communities. (E) Among Gram-negative bacteria, Verrucomicrobia (Akkermansia muciniphila) was increased by 9 -fold (percentage of total bacteria). These changes were prevented by concurrent feeding with $\mathrm{CHO}$. ( $n=6-8$ per group). CHO, cholestyramine. $P$ values were determined by 1 -way ANOVA test. ${ }^{*} P<0.05$ versus RC; ${ }^{\#} P<0.05$ versus HFD. 
A

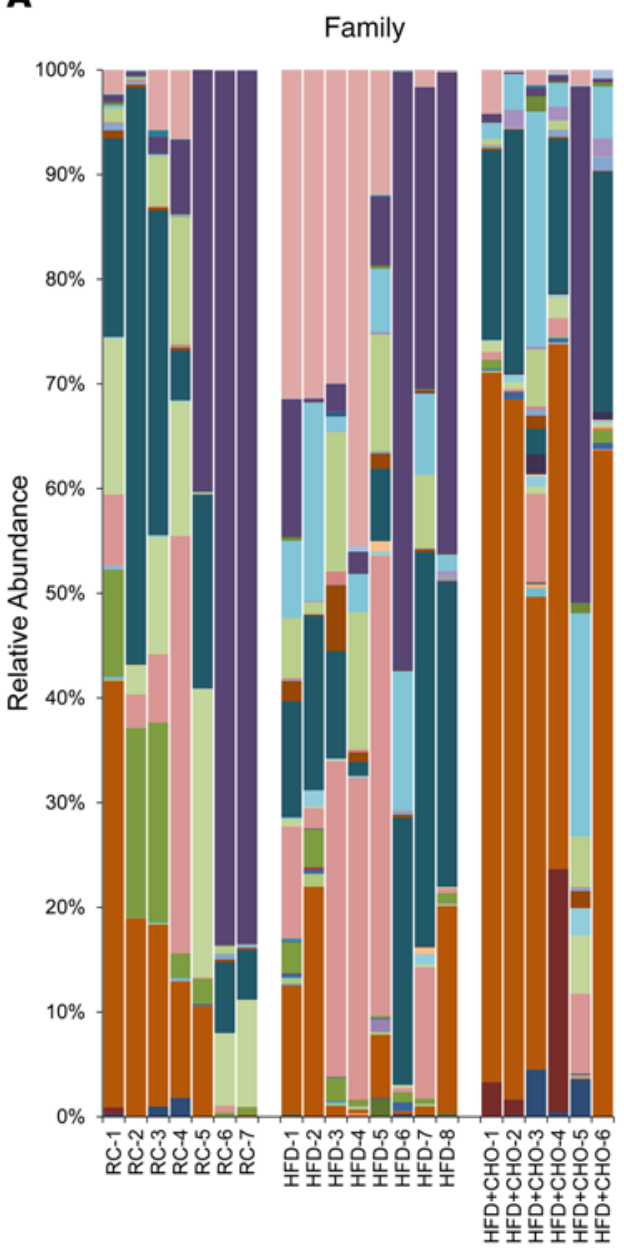

= Xanthomonadaceae Verrucomicrobiaceae Veillonellaceae

- Unclassified Verrucomicrobia - Unclassified Proteobacteria - Unclassified Firmicutes

Unclassified Bacteroidetes - Unclassified Actinobacteria

- Sutterellaceae

- Streptomycetaceae

= Streptococcaceae

Staphylococcaceae

Ruminococcaceae

Rikenellaceae

- Prevotellaceae

- Porphyromonadaceae

Peptostreptococcaceae

- Pasteurellaceae

- Paenibacillaceae

- Oxalobacteraceae

- Neisseriaceae

Moraxellaceae

- Micrococcaceae

- Leptotrichiaceae

Lactobacillaceae

= Lachnospiraceae

Helicobacteraceae

- Fusobacteriaceae

- Flavobacteriaceae

- Eubacteriaceae

- Erysipelotrichaceae

- Enterococcaceae

- Enterobacteriaceae

- Desulfovibrionaceae

- Deferribacteraceae

- Corynebacteriaceae

= Coriobacteriaceae

- Comamonadaceae

- Clostridiales Incertae Sedis XI

- Clostridiaceae

- Caulobacteraceae

- Campylobacteraceae

- Burkholderiaceae

Bifidobacteriaceae

- Bacteroidaceae

- Bacillales Incertae Sedis XI

= Bacillaceae

Anaeroplasmataceae

- Aerococcaceae

- Actinomycetaceae

acidaminococcaceae
D

Lactobacillaceae (Lactobacillus spp.)

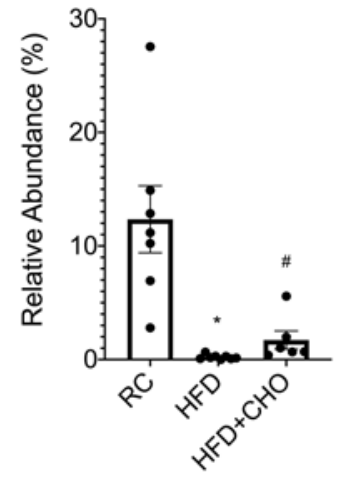

Verrucomicrobiaceae (Akkermansia sp.)

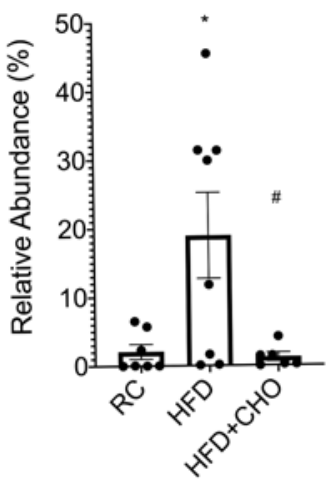

B

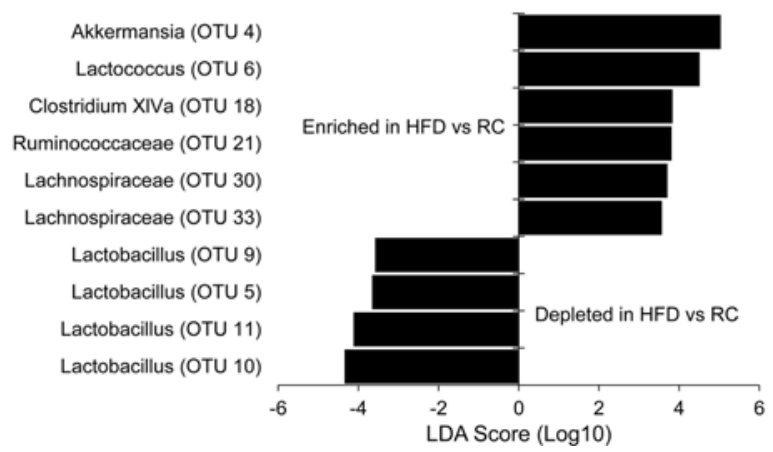

\section{C}

Clostridium sensu stricto (OTU 2)

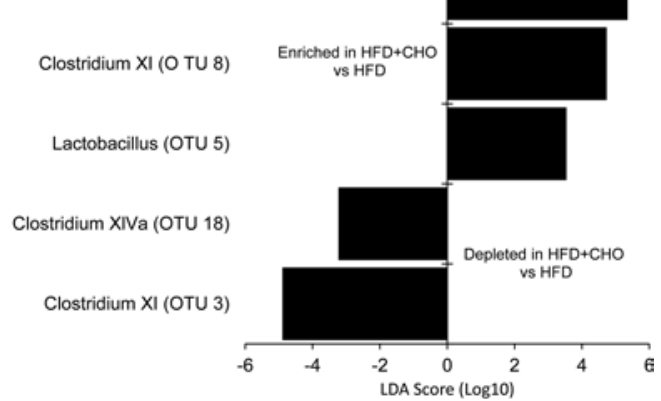

Figure 6. HFD-induced dysbiosis in the ileum mucosa is prevented by cholestyramine. (A) Relative abundance of OTUs classified at the level of family. (B and C) OTUs were significantly depleted or enriched after HFD (LEfSe, $n=7$ or 8 per group). (D) As a group, the relative abundance of Lactobacillaceae and Verrucomicrobiaceae was altered after HFD+CHO compared with HFD ( $n=6$ or 8 per group). $P$ values were determined by unpaired 2-tailed Student's $t$ test ( 2 groups) or by 1 -way ANOVA (more than 2 groups). ${ }^{*} P<0.05$ versus $\mathrm{RC} ;{ }^{*} P<0.05$ versus $\mathrm{HFD}$. CHO, cholestyramine.

probably more important for nutrient digestion, we analyzed changes in the mucosal-associated microbiota, which are intimately associated with the host epithelium and are likely more relevant in studies of host-microbial immune signaling (31) and mucosa permeability. We noted that Akkermansia muciniphila, a genus in the phylum of Verrucomicrobia, was significantly increased following HFF (Figure 5B). It is well known that HFF is associated with impaired intestinal permeability and mucosal inflammation (32, 33). Akkermansia muciniphila is a Gram-negative anaerobe and a mucin degrader that uses mucin as nutrients. Thinning of the mucus layer 
A
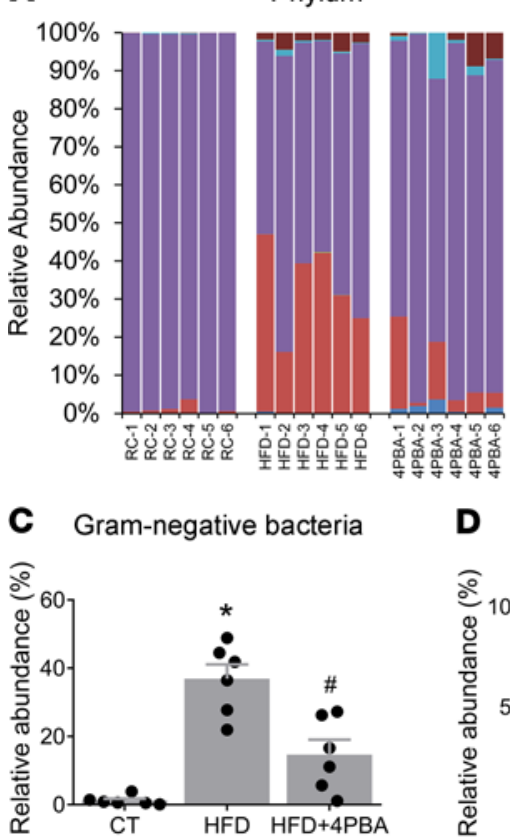

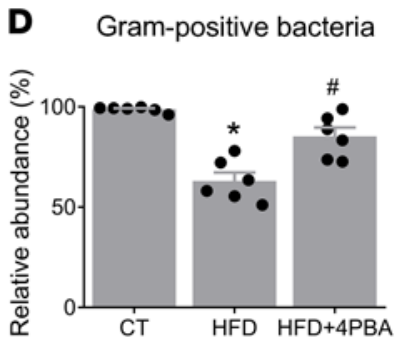

B Firmicutes

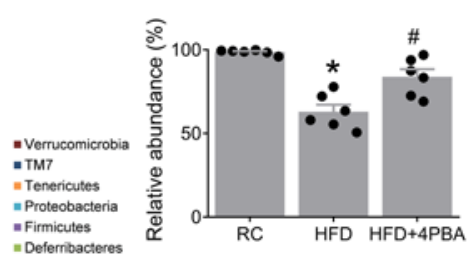

B Firmicutes

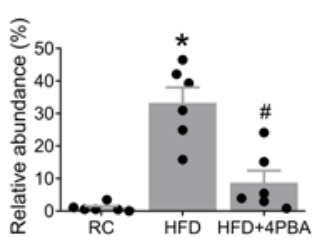

Verucomicrobia

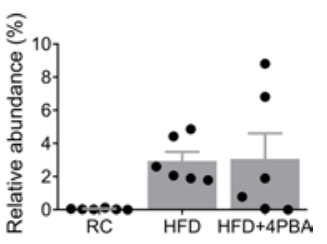

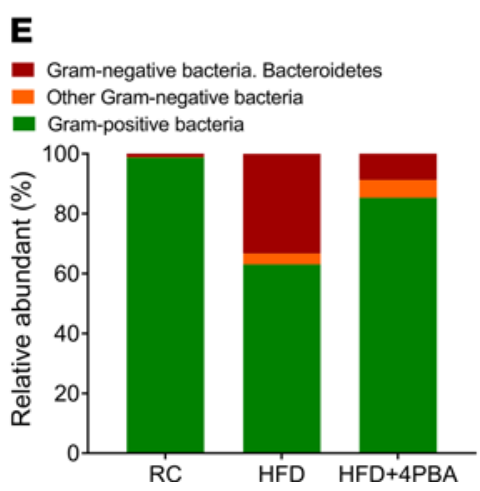

Figure 7. HFD-induced dysbiosis at phylum level in the ileum mucosa is prevented by 4PBA. (A) Relative abundance of OTUs classified at the level of phylum. (B) Relative abundance of Firmicutes, Bacteroidetes, and Verrucomicrobia. (C and D) Relative abundance of (C) Gram-negative and (D) -positive communities. (E) Among Gram-negative bacteria, Bacteroidetes was increased by 31.62-fold, from 1.05\% $\pm 0.50 \%$ to $33.28 \% \pm 4.75 \%$ (percentage of total bacteria) ( $n=6$ per group). These changes were prevented by 4PBA (ER stress inhibitor). $P$ values were determined by 1 -way ANOVA. ${ }^{*} P<0.05$ versus RC; ${ }^{\#}<0.05$ versus HFD.

may weaken gut barrier function and enhance transepithelial migration of microbes and their metabolites. This, in turn, predisposes the host to develop metabolic syndrome, which is common among subjects on a HFD (34).

In addition to phylum-level changes, we also observed depletion of certain OTUs, such as Lactobacillus, while certain OTUs were enriched, including Clostridium XIVa group, Ruminococcaceae, Lachnospiraceae, and Akkermansia muciniphila in rats receiving HFD. These changes are likely due to dysfunction of the Paneth cell; however, local factors may also contribute to alterations in microbial composition in the ileum. The mucosal microenvironment may favor specific microorganisms, including known butyrate producers Clostridium XIVa cluster, Ruminococcaceae, and Lachnospiraceae families, compared with the luminal space $(31,35)$. Moreover, the presence of bile acids places a major selective pressure on the microbial community structure in the gut, with Gram-negative bacteria showing higher tolerance to bile acids compared with Gram-positives bacteria (12). Prior studies have demonstrated that diets supplemented with CA-enriched bacteria possessing $7 \alpha$-dehydroxylation activity, including members of Clostridium XIVa group, Ruminococcaceae, and Lachnospiraceae $(7,9)$. Another study showed a significant and positive correlation between secondary bile acid concentration in feces and abundance of members of the Clostridium XIVa cluster (36). Our results also support this and suggest that HFD and bile acids promote expansion of the Clostridium XIVa cluster. Meanwhile, Floch et al. reported that bile acids appear to be toxic to Lactobacillus in a dose-dependent manner (37). Reduction in the abundance of Lactobacillus, a major source of bile salt hydrolase activity in the gut, may result in increased tauro- $\beta$-muricholic acid levels, inhibition of intestinal FXR signaling, and further increased bile acid pool size (38). Taken collectively, this suggests that a HFD results in increased bile acids, which may cause ileal dysbiosis via direct microbial actions as well as inducing Paneth cell dysfunction.

To demonstrate that taxonomic shifts are mediated by bile acids, rats were fed either a diet of RC supplemented with CA or a HFD mixed with $\mathrm{CHO}$ to decrease the bile acid pool $(39,40)$. Murine animals fed normal chow mixed with CA demonstrated similar taxonomic shifts as those provided with a HFD $(7,9)$. In contrast, administration of $\mathrm{CHO}$ along with a HFD to rats resulted in normalization of microbial composition similar to that of rats receiving RC. Specifically, Gram-positive and Gram-negative bacteria levels normalized as did the relative abundance of Firmicutes and Verrucomicrobia and Lactobacillus and Clostridium XIVa cluster after administration of $\mathrm{CHO}$. These data support the notion that bile acids promote changes in the gut microbial ecology and that these changes can be reversed by administration of a bile acid sequestrant. 
A

A

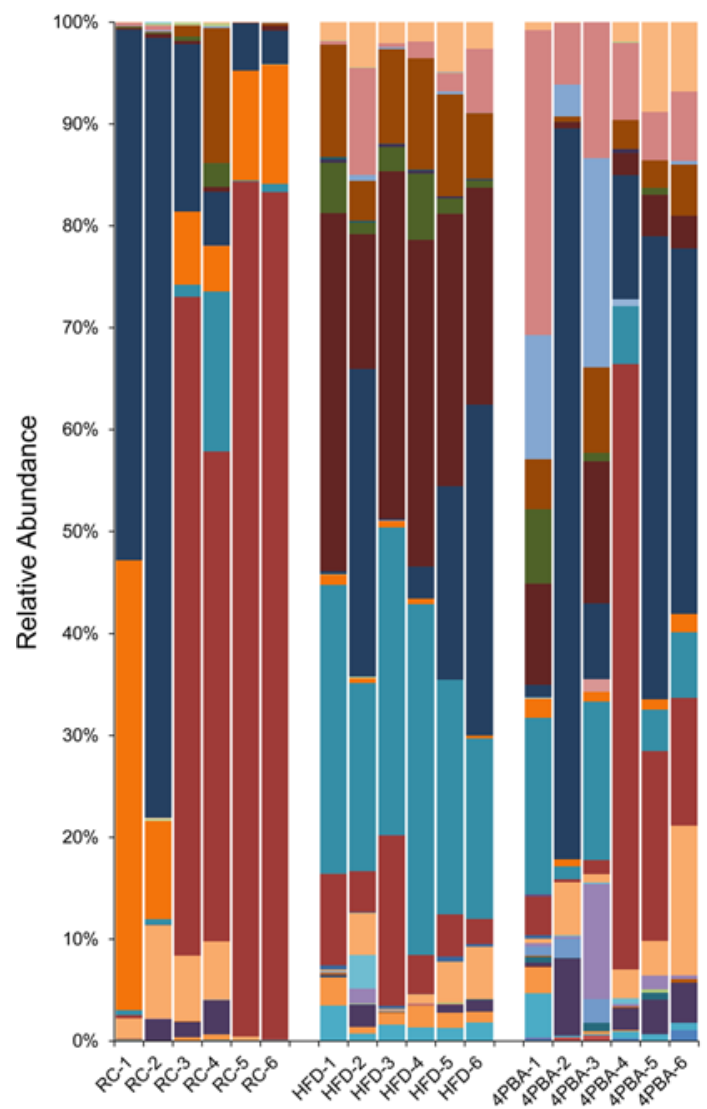

= Verrucomicrobiaceae

- Veillonellaceae

TM7 family incertae sedis

Sutterellaceae

= Streptococcaceae

Staphylococcaceae

- Ruminococcacea

- Rikenellaceae

- Proteobacteria Unclassified

- Prevotellaceae

- Porphyromonadaceae

- Peptostreptococcaceae

= Paenibacillaceae

Mycoplasmataceae

Moraxellaceae

Inicrococcaceae

= Microbacteriaceae

= Leuconostocaceae

- Lactobacillaceae

- Lachnospiraceae

- Helicobacteraceae

- Flavobacteriaceae

- Firmicutes Unclassified

- Eubacteriaceae

Erysipelotrichaceae

Enterococcaceae

= Enterobacteriaceae

- Desulfovibrionaceae

- Deferribacteraceae

Corynebacteriaceae

- Coriobacteriaceae

- Comamonadaceae

- Clostridiaceae

- Caulobacteraceae

- Burkholderiaceae

- Bifidobacteriaceae

- Bacteroidetes Unclassified

- Bacteroidaceae

- Bacillaceae

- Anaeroplasmataceae

- Aerococcaceae

- Actinobacteria Unclassified
D Porphyromonadaceae
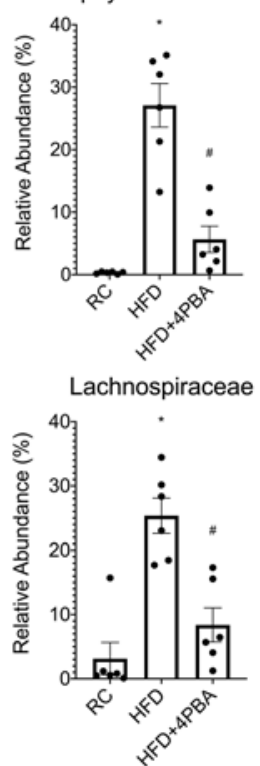

Ruminococcaceae

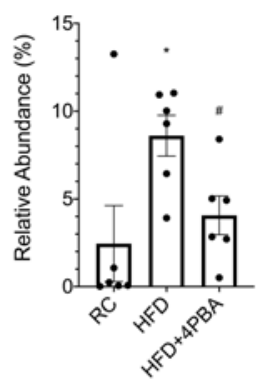

B

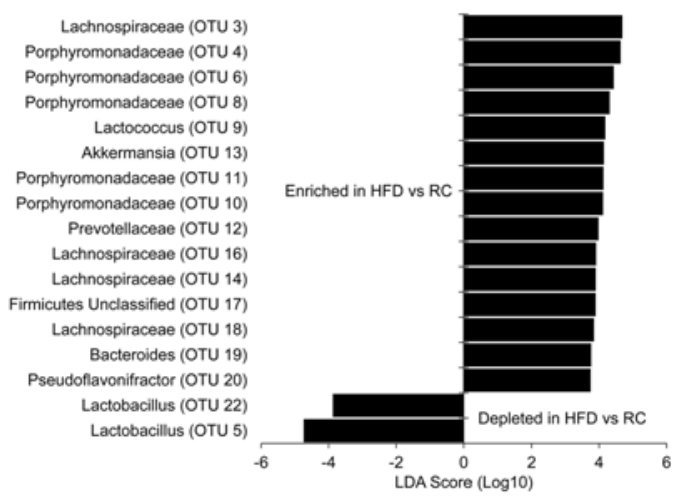

C

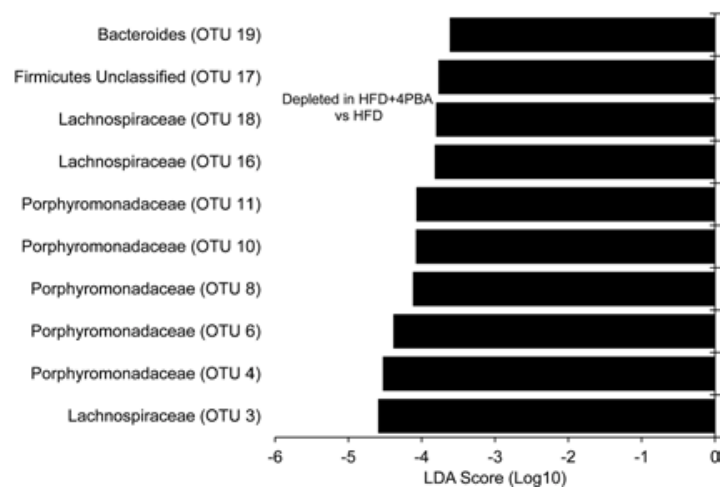

Figure 8. HFD-induced dysbiosis in the ileum mucosa is prevented by 4PBA. (A) Relative abundance of OTUs classified at the level of family. (B and C) OTUs were significantly depleted or enriched after HFD (LEfSe, $n=6$ per group). (D) As a group, the relative abundance of Porphyromonadaceae, Lachnospiraceae, and Ruminococcaceae was altered after HFD+4PBA compared with HFD. $P$ values were determined by unpaired 2-tailed Student's $t$ test (2 groups) or by 1 -way ANOVA (more than 2 groups). ${ }^{*} P<0.05$ versus RC; ${ }^{*} P<0.05$ versus HFD.

It is interesting that the bile acid binder $\mathrm{CHO}$ produced only a modest recovery of Lactobacilli following HFF. This may be due to the fact that Lactobacilli are highly sensitive to the inhibitory actions of bile acids. Both DCA and chenodeoxycholic acid show inhibitory effects on Lactobacilli at a concentration as low as $0.67 \mathrm{mM}$ (37). Hence, the small amount of bile acids remaining in the lumen that are not bound by CHO may be sufficient to inhibit the growth of Lactobacilli.

Murakami et al. reported that a HFD resulted in increased colonic bile acid concentration and provided evidence of epithelial barrier dysfunction (41). Gut dysbiosis likely causes this permeability impairment secondary to Paneth cell dysfunction. In the presence of HFD or RC supplemented with 
A

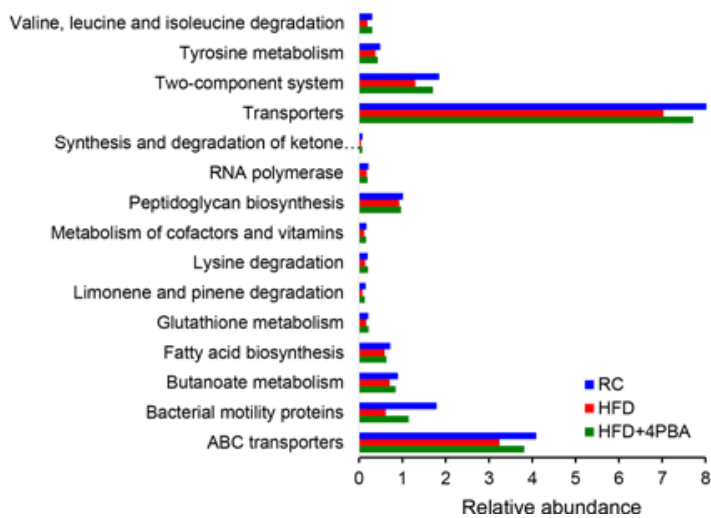

B

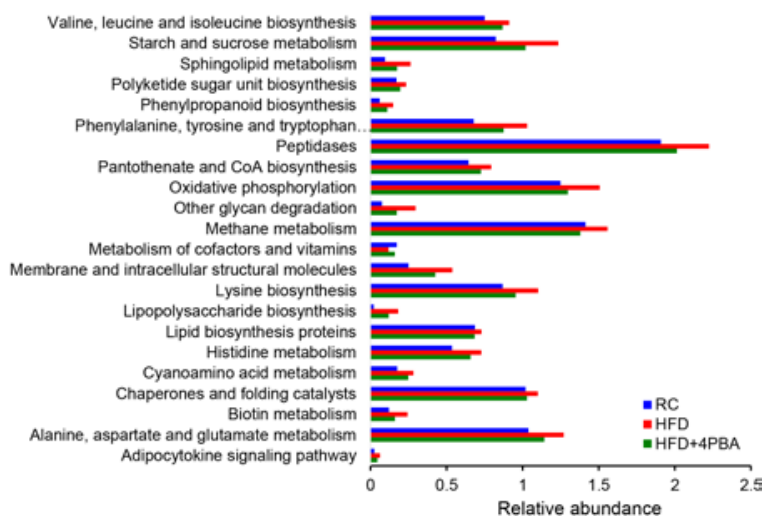

Figure 9. HFD altered genes from microbial community, which was prevented by 4PBA. (A) The relative abundance of metabolic gene pathways was altered in HFD group. The abundance of metabolic gene pathways was analyzed using PICRUSt and is based on the 16S rRNA sequencing data. Only the relative gene pathway abundances that were identified as being significantly different for RC, HFD, and HFD+4PBA are shown $(P<0.01$, for all pairwise comparisons, by 1-way ANOVA). Note that, genes for Gram-positive cell wall synthesis (peptidoglycan biosynthesis) were decreased in the HFD group, and this was reversed by 4PBA treatment. (B) Compared with the regular chow (RC), HFD increased many genes, mainly, for amino acid and energy metabolism. Genes responsible for synthesis of the Gram-negative cell wall (lipopolysaccharide biosynthesis, lipopolysaccharide biosynthesis proteins) were enriched in the HFD group, and this was also reversed by 4PBA.

CA, there is upregulation of TGR5 expression and evidence of Paneth cell dysfunction marked by loss of secretory granules, decreased expression of $\alpha$-defensins, and markers of ER stress and autophagy Paneth cells are known to directly sense and regulate host-microbial interactions through MyD88dependent Toll-like receptor activation (24). Activation of Paneth cells results in release of antimicrobial peptides that promote integrity of the gut epithelium. However, Paneth cells only sense bacteria closely associated with the mucosal surface; they are relatively insensitive to luminal bacterial populations. This may be due to the fact that antimicrobial factors secreted by Paneth cells are largely compartmentalized within the mucus layer covering the intestinal epithelium (42). These observations may likely explain the differential effects of bile acids on mucosal versus luminal bacteria composition in the ileum.

We next performed ex vivo studies to provide evidence that bile acids act directly on Paneth cells. Incubation of ileal explants with DCA or CA resulted in a significant decrease in gene expression of Defa5 and Defa6. Similarly, prior studies have also reported decreased expression of antimicrobial peptides by Paneth cells in response to HFD. Guo et al. demonstrated that HFD in mice resulted in alteration in the gut microbial composition accompanied by reduced antimicrobial peptides lysozyme and Reg III $\gamma$ (43). Similarly, Lee and colleagues reported that mice fed a HFD had decreased Paneth cells, reduced lysozyme release, and disruption of intestinal barrier function and were more susceptible to experimentally induced colitis (44). These data suggest that a HFD is detrimental to Paneth cell function, which results in reduced antimicrobial peptide release. Our study demonstrates that harmful action of HFD is mediated by bile acids acting directly on Paneth cells.

In order to determine the mechanisms by which bile acids induce Paneth cell injury and dysfunction, we demonstrated that HFF significantly increased gene expression of markers of ER stress response (XBP-1) as well as a marker of autophagy (ATG16L1). We confirmed that upregulation of gene expression was occurring in Paneth cells by immunohistochemistry. Protein expression of ER stress marker ( $\mathrm{BiP}$ ) was enhanced in vivo when rats were fed $\mathrm{RC}$ supplemented with $\mathrm{CA}$, and gene expression of XBP-1 was upregulated in ileal explants when they were cultured in the presence of DCA or CA. On the other hand, gene expression normalized when rats were fed a HFD along with CHO. Similarly, gene expression of XBP-1 was normalized in vitro in the presence of TGR5 antibodies or 4PBA. Moreover, we also showed that inhibition of ER stress response by 4PBA could reverse community-level changes in gut microbial composition. This suggests that bile acid-induced Paneth cell dysfunction plays a major role in gut dysbiosis in the ileal mucosa induced by HFD. By virtue of their highly secretory function, Paneth cells are characterized by extensive networks of ER and thus are particularly susceptible to increased ER stress response and autophagy (45). Prior reports have demonstrated that a HFD and obesity induce ER stress in different organ systems, including adipose tissue, the amygdala, and the 
thyroid gland (46-48). Guo et al. reported increased mRNA expression of ER stress markers, including $B i P$, activating transcription factor 4 (ATF4), and c/EBP-homologous protein $(C H O P)$ in mice fed a HFD (49). Meanwhile, Hodin et al. demonstrated an inverse correlation between Paneth cell antimicrobial peptides and measures of ER stress, such as BiP and ATF4 levels, in obese individuals (27). Taken collectively, these data indicate that HFD has detrimental effects on Paneth cells. Our studies demonstrated that these abnormalities are mediated by the TGR 5 receptor, which activates ER stress responses and autophagy. These events result in Paneth cell dysfunction, reduced expression of antimicrobial peptides, and subsequent gut dysbiosis in the ileal mucosa. Furthermore, this compositional shift in the gut microbial microenvironment is characterized by overabundance of certain taxa that are geared toward increasing the bile acid pool size, which may result in further deleterious changes in Paneth cell function. Although bile acids may also act via the bile acid nuclear receptor (FXR), we demonstrated that FXR agonists do not affect gene expression of antimicrobial peptides by Paneth cells. This suggests that TGR5 is the main pathway by which HFF induces alterations in Paneth cell function.

A defect in Paneth cell numbers and antimicrobial peptide production has been found in certain gastrointestinal disorders, such as Crohn's disease $(50,51)$ and necrotizing enterocolitis $(52)$, leading to intestinal dysbiosis. Recently, Günther and colleagues (53) reported that patients with Crohn's disease had significant increases in interferon $\lambda$, accompanied by severe ileal inflammation and loss of Paneth cells. In Crohn's disease, the decline of Paneth cell appears to be associated with caspase-8mediated programmed necrosis (53). On the other hand, in our studies we observed that caspase-3 was increased following HFF or oral feeding with CA. This suggests that apoptosis is a major pathway leading to the loss of Paneth cells in bile acid toxicity. We did not examine caspase- 8 and cannot rule out its participation in the decrease of Paneth cell following HFF.

Our studies have some limitations. To provide conclusive evidence that bile acid toxicity on Paneth cells contributes to gut dysbiosis we need a Paneth cell-specific TGR5-knockout rodent model. In the absence of such a genetic model, we demonstrated that the bile acid binder $\mathrm{CHO}$ reversed the effects on gut dysbiosis induced by HFF. This suggests that ileal mucosal dysbiosis is likely mediated directly or indirectly by bile acids. We also showed that an inhibitor of ER stress given systemically prevented dysbiosis following HFF, suggesting that alteration in ileal microbial composition may result from metabolic malfunctioning of the host immune system, such as Paneth cells. While this interpretation is logical, it may be subject to criticism. $\mathrm{CHO}$ is an anion exchange resin that binds more than just bile acids. The ER stress inhibitor 4PBA may act on a number of cell types other that Paneth cells. Hence, further studies using tissue-specific TGR5-knockout models are needed to confirm our hypothesis.

In conclusion, we propose that HFF leads to increased bile acid production and upregulation of TGR5 receptors in Paneth cells. Overactivation of TGR5 by bile acids causes ER stress, resulting in autophagia and reduction of secretion of defensins into the intestinal crypts. This creates an environment favoring the growth of Gram-negative bacteria adjacent to the ileal epithelium. Paneth cell injury can be prevented by inhibition of ER stress, such as the use of 4PBA, to restore normal production of defensins and prevents dysbiosis occurring adjacent to the ileal mucosa (Figure 10). Further studies may focus on TGR5 as a potential therapeutic target for obesity and metabolic-related conditions.

\section{Methods}

\section{Materials}

The chemicals used in this study are described in detail in the Supplemental Methods (supplemental material available online with this article; https://doi.org/10.1172/jci.insight.138881DS1).

\section{Animal preparation}

Experiments were performed on male Sprague-Dawley rats (100-150 g) (Charles River Laboratories). Rats were housed 3 per cage in a pathogen-free facility maintained on a 12-hour light/dark cycle. They were given food and water ad libitum and allowed to acclimate to the facility for 2 days before being randomly assigned to dietary treatments.

Rats were fed for 2 consecutive weeks with the following: (a) a control diet of RC (11.3\% kcal from fat), (b) a HFD (58\% kcal from fat, D12330, Research Diets), (c) a HFD mixed with 6\% (w/w) CHO (bile acid binder C4650, MilliporeSigma), or (d) RC mixed with CA (0.051\% C1129, MilliporeSigma). 
A

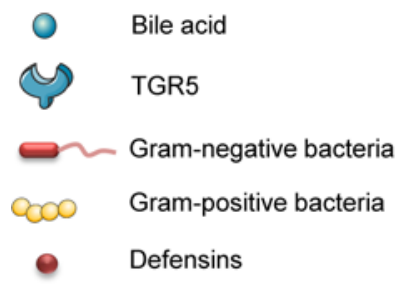

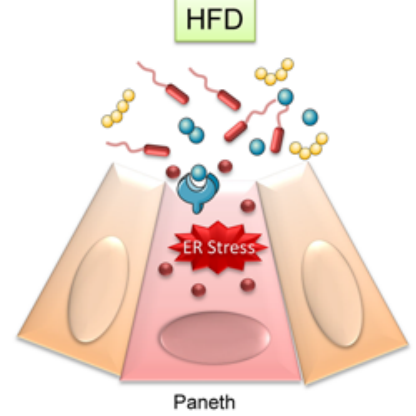

B

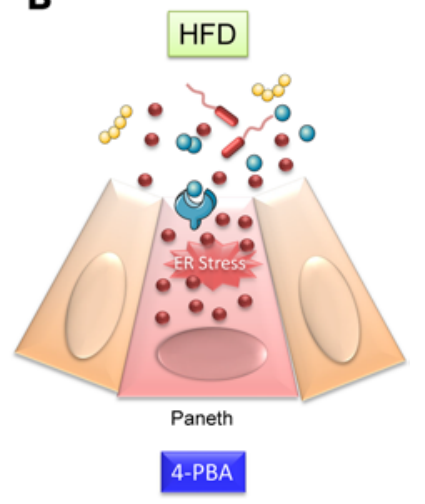

Figure 10. A schematic diagram depicting the mechanism by which bile acids cause gut dysbiosis through Paneth cells. (A) HFD lead to increased bile acid production and high concentrations of DCA. The activation of TCR5 by DCA causes ER stress, autophagy, and reduction of $\alpha$-defensins in Paneth cell, which favors the growth of Gram-negative bacteria and results in mucosal microbial dysbiosis. (B) These changes are prevented by 4PBA.

In a separate group, rats were fed for 2 consecutive weeks with the following: (a) a control diet of RC, (b) a HFD, (c) HFD+4PBA (MilliporeSigma) (100 mg/kg/d, i.p.).

At the end of the 2-week period, rats in each group were food deprived for 12 hours and then euthanized. Fresh serum and fecal samples were collected after a 12 -hour fast and stored at $-80^{\circ} \mathrm{C}$ for analysis of bile acid concentration. Ileal tissue and crypts were harvested for immunochemistry, flow cytometry, MiSeq analysis of bacteria, Western blot, RT-qPCR, electron microscopy and ileal explant culture studies.

\section{Total bile acid concentration measurement}

Total bile acid from fecal samples and serum were measured enzymatically using the Rat Total Bile Acids Assay Kit (Crystal Chem). Fecal samples were homogenized in 75\% ethanol and incubated for 2 hours at $50^{\circ} \mathrm{C}$. The extract was centrifuged at $13,000 \mathrm{~g}$ for 10 minutes, and fecal supernatant was loaded for determination of absorbance at $540 \mathrm{~nm}$, according to the manufacturer's instruction $(54,55)$.

\section{Illumina MiSeq analysis of bacteria}

MiSeq Illumina sequencing. Samples were processed using the MiSeq Illumina sequencing platform. 16S rRNA gene libraries were constructed using primers specific to the V4 region (see Supplemental Methods).

OTU assignment. Sequences were curated using mothur (v.1.40) (56) following the steps outlined in the MiSeq SOP (57). Sequences were assigned to OTUs using a $97 \%$ similarity cutoff and classified against the Ribosomal Database Project 16S rRNA gene training set (version 16) using a naive Bayesian approach with an $80 \%$ confidence threshold. Curated OTU sequence data were converted to relative abundance \pm standard error of the mean. To test for OTUs that were differentially abundant, biologically consistent, and having the greatest effect size, we used LEfSe (58). Only OTUs with a relative abundance $>0.5 \%$ were considered in the LEfSe analyses.

\section{Prediction of the bacterial metagenome from the ileum mucosa}

To predict functional potential, and better understand the biological significance of alterations in mucosal microbial communities induced by HFD and 4PBA, we analyzed the relationship between microbial structure and function associated with HFF with or without 4PBA using PICRUSt (59).

\section{Epithelial cell isolation, Western blot, and RT-PCR}

Crypts were isolated from terminal ileum as previously described by Sato et al. (60) with some modifications. Crypts were isolated and used for RT-qPCR or Western blot studies to examine gene and protein expression of antimicrobial peptides, Defa5 and Defa6; markers of ER stress, including XBP-1, and autophagy, including ATG16L1; and poly ADP ribose polymerase (PARP). For further details, see the Supplemental Methods.

\section{Immunohistochemistry}

Sections of ileal tissue and isolated epithelial cells were used for immunohistochemistry studies as described previously (see the Supplemental Methods) (61). 
In situ hybridization

Sections of ileal tissue were used for in situ hybridization studies as described previously (see the Supplemental Methods) (62).

Flow cytometry

Epithelial cells were isolated as described above and then underwent flow cytometry studies as previously described (see the Supplemental Methods) (63).

\section{Electron microscopy}

Detailed procedures for electron microscopy have been previously described (28) (see the Supplemental Methods).

Ex vivo study of ileal explant culture

To provide direct evidence that bile acids can act directly to affect Paneth cells, ileal explants were incubated with DCA $(100 \mu \mathrm{M})$ or CA $(100 \mu \mathrm{M})$ for 24 hours with or without TGR5 antibody or ER stress inhibitor (4PBA) to determine gene expression of $\alpha$-defensins and XBP-1 (ER stress markers) (see the Supplemental Methods).

\section{Statistics}

All results are shown as mean \pm SEM, with the number of rats indicated. For statistical analyses, differences between groups were compared by 2-tailed Student $t$ test for comparisons between 2 groups or 1-way ANOVA for comparisons of more than 2 groups. $P<0.05$ was considered significant.

\section{Study approval}

All procedures were performed in accordance with the NIH guidelines and were approved by the University of Michigan Committee on Use and Care of Animals.

\section{Author contributions}

CO conceived and designed research. HZ, SYZ, MG, JYL, JG, GZ, and XX obtained data from the study. HZ, SYZ, MG, and JYL analyzed and interpreted the data. HZ, SYZ, AL, and CO wrote the manuscript. $\mathrm{CO}$ and $\mathrm{HZ}$ obtained funding.

\section{Acknowledgments}

The studies were supported by NIH grants R01 DK058913 and P30 DK34933 and National Natural Science Foundation of China grant 81570475.

Address correspondence to: Chung Owyang, 3912 Taubman Center, SPC 5362, Division of Gastroenterology and Hepatology, Department of Internal Medicine, University of Michigan Health System, Ann Arbor, Michigan 48109, USA. Phone: 734.936.4785; Email: cowyang@med.umich.edu.

1. Albenberg LG, Wu GD. Diet and the intestinal microbiome: associations, functions, and implications for health and disease Gastroenterology. 2014;146(6):1564-1572.

2. Graf D, et al. Contribution of diet to the composition of the human gut microbiota. Microb Ecol Health Dis. 2015;26:26164

3. Llewellyn SR, et al. Interactions between diet and the intestinal microbiota alter intestinal permeability and colitis severity in mice. Gastroenterology. 2018;154(4):1037-1046.e2.

4. Martinez-Medina M, et al. Western diet induces dysbiosis with increased E coli in CEABAC10 mice, alters host barrier function favouring AIEC colonisation. Gut. 2014;63(1):116-124.

5. Just S, et al. The gut microbiota drives the impact of bile acids and fat source in diet on mouse metabolism. Microbiome. 2018;6(1):134.

6. Reddy BS, Mangat S, Sheinfil A, Weisburger JH, Wynder EL. Effect of type and amount of dietary fat and 1,2-dimethylhydrazine on biliary bile acids, fecal bile acids, and neutral sterols in rats. Cancer Res. 1977;37(7 Pt 1):2132-2137.

7. Zheng X, et al. Bile acid is a significant host factor shaping the gut microbiome of diet-induced obese mice. BMC Biol. 2017; 15(1):120.

8. Devkota S, Chang EB. Interactions between diet, bile acid metabolism, gut microbiota, and inflammatory bowel diseases. Dig Dis. 2015;33(3):351-356.

9. Islam $\mathrm{KB}$, et al. Bile acid is a host factor that regulates the composition of the cecal microbiota in rats. Gastroenterology. 2011;141(5):1773-1781.

10. Xiong F, et al. Dietary bile salt types influence the composition of biliary bile acids and gut microbiota in grass carp. Front Microbiol. 2018;9:2209. 
11. Ridlon JM, Alves JM, Hylemon PB, Bajaj JS. Cirrhosis, bile acids and gut microbiota: unraveling a complex relationship. Gut Microbes. 2013;4(5):382-387.

12. Ridlon JM, Harris SC, Bhowmik S, Kang DJ, Hylemon PB. Consequences of bile salt biotransformations by intestinal bacteria. Gut Microbes. 2016;7(1):22-39.

13. Tsuei J, Chau T, Mills D, Wan YJ. Bile acid dysregulation, gut dysbiosis, and gastrointestinal cancer. Exp Biol Med (Maywood). 2014;239(11):1489-1504.

14. Bernstein H, Payne CM, Bernstein C, Schneider J, Beard SE, Crowley CL. Activation of the promoters of genes associated with DNA damage, oxidative stress, ER stress and protein malfolding by the bile salt, deoxycholate. Toxicol Lett. 1999;108(1):37-46.

15. Wang H, Chen J, Hollister K, Sowers LC, Forman BM. Endogenous bile acids are ligands for the nuclear receptor FXR/BAR. Mol Cell. 1999;3(5):543-553.

16. Maruyama T, et al. Identification of membrane-type receptor for bile acids (M-BAR). Biochem Biophys Res Commun. 2002;298(5):714-719.

17. Kawamata Y, et al. A G protein-coupled receptor responsive to bile acids. J Biol Chem. 2003;278(11):9435-9440.

18. Marin JJ, Macias RI, Briz O, Banales JM, Monte MJ. Bile Acids in Physiology, Pathology and Pharmacology. Curr Drug Metab. 2015;17(1):4-29.

19. Chen X, Lou G, Meng Z, Huang W. TGR5: a novel target for weight maintenance and glucose metabolism. Exp Diabetes Res. 2011;2011:853501.

20. Poole DP, et al. Expression and function of the bile acid receptor GpBAR1 (TGR5) in the murine enteric nervous system. Neurogastroenterol Motil. 2010;22(7):814-825, e227.

21. Pols TW, Noriega LG, Nomura M, Auwerx J, Schoonjans K. The bile acid membrane receptor TGR5 as an emerging target in metabolism and inflammation. J Hepatol. 2011;54(6):1263-1272.

22. Pang C, et al. Bile salt receptor TGR5 is highly expressed in esophageal adenocarcinoma and precancerous lesions with significantly worse overall survival and gender differences. Clin Exp Gastroenterol. 2017;10:29-37.

23. Cao W, et al. Expression of bile acid receptor TGR5 in gastric adenocarcinoma. Am J Physiol Gastrointest Liver Physiol. 2013;304(4):G322-G327.

24. Vaishnava S, Behrendt CL, Ismail AS, Eckmann L, Hooper LV. Paneth cells directly sense gut commensals and maintain homeostasis at the intestinal host-microbial interface. Proc Natl Acad Sci USA. 2008;105(52):20858-20863.

25. Clevers HC, Bevins CL. Paneth cells: maestros of the small intestinal crypts. Annu Rev Physiol. 2013;75:289-311.

26. Zhang Z, Liu Z. Paneth cells: the hub for sensing and regulating intestinal flora. Sci China Life Sci. 2016;59(5):463-467.

27. Hodin CM, et al. Reduced Paneth cell antimicrobial protein levels correlate with activation of the unfolded protein response in the gut of obese individuals. J Pathol. 2011;225(2):276-284.

28. Ley RE, Bäckhed F, Turnbaugh P, Lozupone CA, Knight RD, Gordon JI. Obesity alters gut microbial ecology. Proc Natl Acad Sci USA. 2005;102(31):11070-11075.

29. Lecomte $\mathrm{V}$, et al. Changes in gut microbiota in rats fed a high fat diet correlate with obesity-associated metabolic parameters PLoS One. 2015;10(5):e0126931.

30. Ohland CL, Jobin C. Microbial activities and intestinal homeostasis: A delicate balance between health and disease. Cell Mol Gastroenterol Hepatol. 2015;1(1):28-40.

31. Van den Abbeele P, Van de Wiele T, Verstraete W, Possemiers S. The host selects mucosal and luminal associations of coevolved gut microorganisms: a novel concept. FEMS Microbiol Rev. 2011;35(4):681-704.

32. Cani PD, et al. Changes in gut microbiota control inflammation in obese mice through a mechanism involving GLP-2-driven improvement of gut permeability. Gut. 2009;58(8):1091-1103.

33. de La Serre CB, Ellis CL, Lee J, Hartman AL, Rutledge JC, Raybould HE. Propensity to high-fat diet-induced obesity in rats is associated with changes in the gut microbiota and gut inflammation. Am J Physiol Gastrointest Liver Physiol. 2010;299(2):G440-G448.

34. Kopp W. How Western diet and lifestyle drive the pandemic of obesity and civilization diseases. Diabetes Metab Syndr Obes. 2019;12:2221-2236.

35. Hill DA, et al. Metagenomic analyses reveal antibiotic-induced temporal and spatial changes in intestinal microbiota with associated alterations in immune cell homeostasis. Mucosal Immunol. 2010;3(2):148-158.

36. Kakiyama G, et al. Modulation of the fecal bile acid profile by gut microbiota in cirrhosis. J Hepatol. 2013;58(5):949-955.

37. Floch MH, Binder HJ, Filburn B, Gershengoren W. The effect of bile acids on intestinal microflora. Am J Clin Nutr. 1972;25(12):1418-1426.

38. Li F, et al. Microbiome remodelling leads to inhibition of intestinal farnesoid X receptor signalling and decreased obesity Nat Commun. 2013;4:2384

39. Angelin B, Björkhem I, Einarsson K, Ewerth S. Cholestyramine treatment reduces postprandial but not fasting serum bile acid levels in humans. Gastroenterology. 1982;83(5):1097-1101.

40. Chiang JY. Bile acid metabolism and signaling. Compr Physiol. 2013;3(3):1191-1212.

41. Murakami Y, Tanabe S, Suzuki T. High-fat diet-induced intestinal hyperpermeability is associated with increased bile acids in the large intestine of mice. J Food Sci. 2016;81(1):H216-H222.

42. Meyer-Hoffert U, et al. Secreted enteric antimicrobial activity localises to the mucus surface layer. Gut. 2008;57(6):764-771.

43. Guo X, et al. High fat diet alters gut microbiota and the expression of Paneth cell-antimicrobial peptides preceding changes of circulating inflammatory cytokines. Mediators Inflamm. 2017;2017:9474896.

44. Lee JC, et al. Obesogenic diet-induced gut barrier dysfunction and pathobiont expansion aggravate experimental colitis. PLoS One. 2017;12(11):e0187515.

45. Kaser A, Blumberg RS. Autophagy, microbial sensing, endoplasmic reticulum stress, and epithelial function in inflammatory bowel disease. Gastroenterology. 2011;140(6):1738-1747.

46. Suzuki T, et al. ER stress protein CHOP mediates insulin resistance by modulating adipose tissue macrophage polarity. Cell Rep. 2017;18(8):2045-2057.

47. Castro G, et al. Diet-induced obesity induces endoplasmic reticulum stress and insulin resistance in the amygdala of rats. FEBS Open Bio. 2013;3:443-449. 
48. Zhang X, et al. ER stress contributes to high-fat diet-induced decrease of thyroglobulin and hypothyroidism. Am J Physiol Endocrinol Metab. 2019;316(3):E510-E518.

49. Guo X, Tang R, Yang S, Lu Y, Luo J, Liu Z. Rutin and its combination with inulin attenuate gut dysbiosis, the inflammatory status and endoplasmic reticulum stress in paneth cells of obese mice induced by high-fat diet. Front Microbiol. $2018 ; 9: 2651$.

50. Wehkamp J, et al. NOD2 (CARD15) mutations in Crohn's disease are associated with diminished mucosal alpha-defensin expression. Gut. 2004;53(11):1658-1664.

51. Wehkamp J, et al. Reduced Paneth cell alpha-defensins in ileal Crohn's disease. Proc Natl Acad Sci USA. 2005;102(50):18129-18134.

52. Coutinho HB, et al. Absence of lysozyme (muramidase) in the intestinal Paneth cells of newborn infants with necrotising enterocolitis. J Clin Pathol. 1998;51(7):512-514.

53. Günther C, et al. Interferon lambda promotes paneth cell death via STAT1 signaling in mice and is increased in inflamed ileal tissues of patients with Crohn's disease. Gastroenterology. 2019;157(5):1310-1322.e13.

54. Han $\mathrm{K}$, et al. In vivo therapeutic effect of combination treatment with metformin and Scutellaria baicalensis on maintaining bile acid homeostasis. PLoS One. 2017;12(9):e0182467.

55. Zhang J, et al. Chronic over-expression of fibroblast growth factor 21 increases bile acid biosynthesis by opposing FGF15/19 action. EBioMedicine. 2017;15:173-183.

56. Schloss PD, et al. Introducing mothur: open-source, platform-independent, community-supported software for describing and comparing microbial communities. Appl Environ Microbiol. 2009;75(23):7537-7541

57. Kozich JJ, Westcott SL, Baxter NT, Highlander SK, Schloss PD. Development of a dual-index sequencing strategy and curation pipeline for analyzing amplicon sequence data on the MiSeq Illumina sequencing platform. Appl Environ Microbiol. 2013;79(17):5112-5120.

58. Segata N, et al. Metagenomic biomarker discovery and explanation. Genome Biol. 2011;12(6):R60.

59. Langille MG, et al. Predictive functional profiling of microbial communities using $16 \mathrm{~S}$ rRNA marker gene sequences. Nat Biotechnol. 2013;31(9):814-821.

60. Sato T, et al. Single Lgr5 stem cells build crypt-villus structures in vitro without a mesenchymal niche. Nature. 2009;459(7244):262-265.

61. Adolph TE, et al. Paneth cells as a site of origin for intestinal inflammation. Nature. 2013;503(7475):272-276.

62. Li JY, et al. Arcuate nucleus transcriptome profiling identifies ankyrin repeat and suppressor of cytokine signalling boxcontaining protein 4 as a gene regulated by fasting in central nervous system feeding circuits. J Neuroendocrinol. 2005;17(6):394-404

63. Gulati AS, et al. Mouse background strain profoundly influences Paneth cell function and intestinal microbial composition. PLoS One. 2012;7(2):e32403. 